\title{
Direct spatial-temporal discrimination of modes in a photonic lightwave circuit using photon scanning tunnelling microscopy
}

\author{
Sam A. Berry, James C. Gates, William S. Brocklesby \\ Optoelectronics Research Centre, University of Southampton, Southampton, United Kingdom, SO17 1BJ
}

Multi-mode photonic lightwave circuits (PLCs) provide new avenues for extending the performance of single mode systems. As an example, they can potentially provide increased bandwidth by multiplexing information into different waveguide modes[1]. For practical applications of multi-mode PLCs to be developed, a measurement technique is required to investigate detailed mode profiles and propagation constants in complex circuits. Photon scanning tunnelling microscopy (PSTM) provides a means of experimentally tracking the femtosecond inter-modal delays observed in PLCs with the ability to discriminate modes by their spatial profiles inside the waveguide.

Using a spectrally-resolved PSTM probe, the guiding properties of multi-mode circuits can be investigated with ultra-high spatial and temporal resolution. In previous work, the relative group velocities of waveguide modes have been measured using a femtosecond laser source and spectral interference[2]. The experiments described here extend previous work by measuring both the spatial profiles and temporal separations of modes using only an incoherent continuous wave $(\mathrm{CW})$ source; thus avoiding unwanted nonlinear effects and simplifying the experimental procedures. To demonstrate the capability, a PSTM probe is used to measure both the spatial and temporal evolution of modes inside a multi-mode $\mathrm{Ta}_{2} \mathrm{O}_{5}$ ridge waveguide. Using a $\mathrm{CW}$ super-luminescent light emitting diode (SLED) light source centered at $\sim 790 \mathrm{~nm}$, the spectrum is measured at each point within the image from which the temporal autocorrelation can be obtained from the Fourier transform. In this simple case, delays build up between the modes due to waveguide dispersion; we exploit this and can determine to which modes the modal delays belong by scrutinising the spatial variations across the guide for a particular delay.
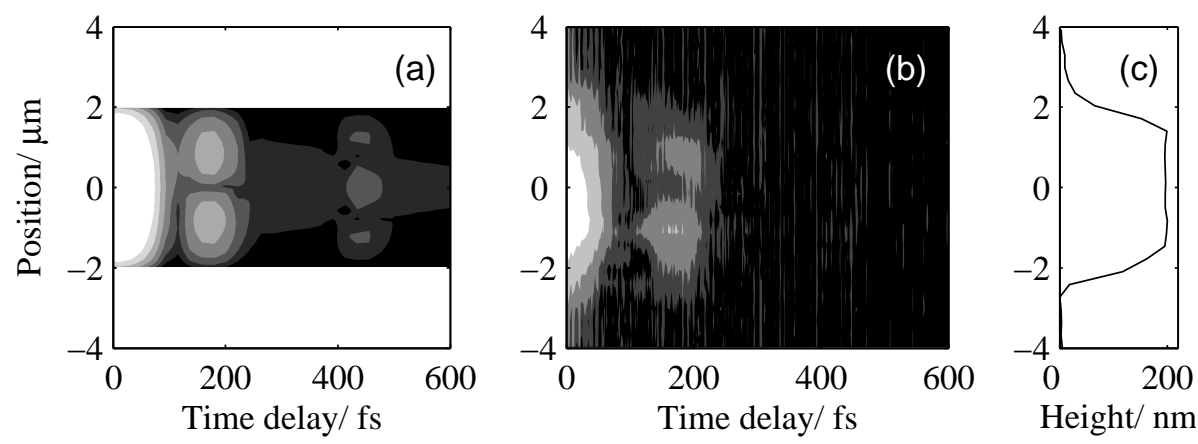

Fig. 1 (a) Theoretical prediction of spatial mode-overlap profiles and respective delays obtained from solving the guidance condition of the $\mathrm{Ta}_{2} \mathrm{O}_{5}$ waveguide using the effective index method for the core region. (b) The measured temporal autocorrelation obtained from spectrally-resolved PSTM probe is plotted as a function of position across the waveguide (log scale intensity contour plot). The mode profiles of the TE00 and TE01 modes can be seen from the temporal separation. (c) The measured height profile plotted as a function of position across the waveguide.

Figure 1(a) shows a theoretical model of the expected inter-modal delays after propagating $8 \mathrm{~mm}$ in the guide, modelled using the effective index method. The $\mathrm{x}$-axis plots the delay between two modes; the y-axis plots the square modulus of the product of the fields of pairs of modes - the spatial mode overlap. Hence the zero time delay signal represents overlap of all modes with themselves, and the feature at $\sim 200 \mathrm{fs}$ delay is the product of the fields of the TE00 and TE01 modes. Figure 1(b) plots the experimental data obtained from $\sim 8 \mathrm{~mm}$ from the input facet of the guide. The spatial profile of the TE00 and TE01 mode overlap is clearly visible, with a temporal separation of $\sim 200 \mathrm{fs}$ between the two. The measured mode overlap profile and position are in good agreement with the calculated values. The theoretical model also displays the delay between the fundamental and second order modes, missing in the data owing to limited signal/noise.

Femtosecond time resolution after propagating light a short distance in waveguides presents PSTM as a promising method for characterising PLCs. The ability to experimentally measure and track the inter-modal delay and spatial profile of modes in PLCs demonstrated here will enable accurate mapping of the propagation and mode structure of light in more complex systems, for example the behaviour of modes at bends and junctions.

\section{References}

[1] J. R. Kurz, J. Huang, X. Xie, T. Saida, and M. M. Fejer “Mode multiplexing in optical frequency mixers,” Optics Letters 29, 551 (2004). [2] J. Mills, T. Chaipiboonwong, W. S. Brocklesby, M. D. B. Charlton, C. Netti, M. E. Zoorob, and J. J. Baumberg "Group velocity measurement using spectral interference in near-field scanning optical microscopy," Appl. Phys. Lett. 89, 51101 (2006). 